\title{
SPH-DEM model for free-surface flows containing solids applied to river ice jams
}

DANIEL M. ROBB, Project Engineer, Northwest Hydraulic Consultants, 30 Gostick Place, North Vancouver, British ColumbiaV7M 3G3, Canada; Email: drobb@nhcweb.com

SUSAN J. GASKIN (IAHR Member), Associate Professor, Department of Civil Engineering and Applied Mechanics, McGill University, Macdonald Engineering Bldg, Room 492, Montréal, QC, Canada H3A 2K6, Canada; Email: Susan.Gaskin@mcgill.ca (author for correspondence) JEAN-CHRISTOPHE MARONGIU, R\&D Engineer, Andritz Hydro, 13 Avenue Albert Einstein 69100 Villeurbanne, France; E-mail: Jean-Christophe.Marongiu@andritz.com

Running Head: SPH-DEM model for river ice jams 


\title{
SPH-DEM model for free-surface flows containing solids applied to river ice jams
}

\begin{abstract}
A meshless method is used to simulate free-surface fluid flows containing solid particles, motivated by the need to simulate river ice dynamics problems. A Smoothed Particle Hydrodynamics model (SPH), with an arbitrary Lagrangian-Eulerian formulation for the fluid phase, is two-way coupled with the Discrete Element Method (DEM) for the solid phase. Validation test cases include a bouncing sphere on a level surface, a collapse of a granular column, wedge entry into still water and solids of different densities falling into still water. The computed results using the SPH-DEM model agree quantitatively with the expected behaviour in the test cases. Numerical convergence is demonstrated for the wedge entry validation case. The SPH-DEM model is then used to simulate the stability of floating ice blocks approaching a stationary cover and ice accumulation upstream of an obstruction. The results show promise to serve as a useful quantitative engineering tool.
\end{abstract}

Keywords: Discrete element method; ice streams; open channel flow; river ice jams; Smoothed Particle Hydrodynamics models

\section{Introduction}

Fluid flows containing solid particles can have complex free surfaces and moving solid boundaries. These features present a challenge for numerical models that use traditional grid-based methods. Meshless methods offer several advantages over grid-based techniques for these types of problems including the accurate description of free surfaces without special treatments such as the volume-of-fluid method, and the automatic tracking of material interfaces without remeshing techniques or sliding grids. Two meshless methods that are particularly well-suited for simulating fluid-particle systems are Smoothed Particle Hydrodynamics (SPH) for the fluid phase and the Discrete Element Method (DEM) for the solid phase. Many problems involving free-surface fluid flow containing solid particles are potential applications for modelling the fluid with SPH and the solid phase with the DEM. Examples include debris flows, landslides, fluidized-bed, wave interaction with armour blocks, and river ice dynamics. Of particular focus in this paper is modelling river ice dynamics problems using a coupled SPH-DEM model.

SPH has been widely used in research to model many free-surface flow problems including dam break waves (Monaghan, 1994), waves breaking on a beach (Dalrymple \& Rogers, 2006), and hydraulic jumps (Lopez, Marivela, \& Garrote, 2010). The DEM has been applied to many environmental and industrial problems involving granular flow including landslides (Cleary \& Campbell, 1993), hopper discharge (Cleary \& Sawley, 2002), floating debris (Stockstill, Daly, \& Hopkins, 2009), and ice floe transport (Gutfraind \& Savage, 1998; Hopkins \& Tuhkuri, 1999; Sun \& Shen, 2012). 
Several studies have used SPH to investigate the motion of a moving solid object in a freesurface flow. Monaghan, Kos, and Issa (2003) was the first to incorporate a moving solid object into an SPH simulation to model a rigid body entering water. Manenti, Panizzo, Ruol, and Martinelli (2008) studied the behaviour of a single floating box in a periodic wave. Rogers, Dalrymple, and Stansby (2010) investigated the movement of a caisson breakwater in the surf zone, modelling both the force exerted on the caisson by periodic wave impact and the friction between the caisson and the bed. Omidvar, Stansby, and Rogers $(2012,2013)$ studied wave body interaction problems in both two and three dimensions using a variable mass particle distribution and an arbitrary Lagrangian-Eulerian (ALE) formulation. The research above successfully used SPH to simulate the motion of a single moving object in a free-surface flow; however, these studies do not consider multiple moving objects experiencing both fluid-solid and solid-solid interactions.

Gotoh, Ikari, and Yasuoka (2009) applied a coupled MPS-DEM model to simulate armour blocks in front of a caisson breakwater. Their study considered multiple moving objects experiencing both fluid-solid and solid-solid interactions in two dimensions. Coupled SPH-DEM models have been applied to fluid-solid systems including slurry flow through industrial screens (Fernandez, Cleary, Sinnott, \& Morrison, 2011), wave interactions with armour blocks on rubble mound breakwaters (Ren, Jin, Gao, Wang, \& Xu, 2013), dam break waves toppling stacked blocks (Canelas, Ferreira, Crespo, \& Domìnguez, 2013), and sedimentation of granular assemblies with various porosities (Robinson, Ramaioli, \& Luding, 2014). Fernandez et al. (2011) used a one-way coupled SPH-DEM model, where there was no feedback from the SPH fluid to the DEM solids. Ren et al. (2013) and Canelas et al. (2013) used two-way coupled SPHDEM models, where the interstitial fluid between solids was modelled with SPH fluid particles. Robinson et al. (2014) presented a two-way coupled SPH-DEM model, where the individual solid particles and the solid-solid interactions were fully resolved. The fluid phase, however, was poorly resolved in the voids between solid particles, which greatly reduces the computational requirements over a fully-resolved model. Instead, the fluid-solid interactions were modelled using a parameterized drag force. The objective of the present study is to enable the accurate simulation of free-surface flows containing solids, resolving both fluid-solid and solid-solid interactions for the purpose of modelling river ice dynamics problems. The approach taken is to use a two-way coupled SPH-DEM model where the fluid is modelled using SPH and the solid particles are modelled using the DEM.

Several static one-dimensional ice jam models have been developed in early studies, including ICEJAM (Flato \& Gerard, 1986), RIVJAM (Beltaos, 1993), and the ice cover/jam routine in HEC-RAS (Hydraulic Engineering Center (HEC), 1998). These models apply the principles of ice jam stability from the pioneering work by Pariset, Hausser, and Gagnon (1966) and Uzuner and Kennedy (1976). While these static ice jam models have had success estimating ice jam thickness and water levels, since dynamic effects are not considered, these models cannot predict whether, when, or where an ice jam will form. Since non-uniform water currents, irregular 
Robb, D., Gaskin, S.J., Marongiu, J-C (2016) SPH-DEM model for free surface flows containing solids applied to river ice jams, Journal of Hydraulic Research, 54(1): 27-40. doi: 10.1080/00221686.2015.1131203

channel geometry, and bank friction make many ice jam processes multi-dimensional, dynamic and two-dimensional ice jam models have been developed, including DynaRICE (Shen, Su, \& Liu, 2000) and SPIKI (Nolin, Roubtsova, Morse, \& Quach, 2009). These river ice models use depth-averaged hydraulic models, which require a much lower computational demand than solving the full internal flow field. Making these simplifying assumptions enables the simulation of considerably larger computational domains. Depth-averaged methods, however, cannot capture the rapidly varying hydraulic conditions that can occur in certain river ice processes, for example, the hydraulics near the leading edge or the toe of an ice jam, ice floe under-turning, jam release, and pile-up. The present study focuses on these smaller scale river ice dynamics processes and makes no attempt to model long river reaches that could be simulated using one of the existing models above.

The river ice models mentioned so far have treated the ice as a continuum. This approach enables modelling large length scales and developing equations of motion describing the overall movement of the ice mass without accounting for the motion of each individual ice fragment. One challenge of the continuum approach is developing appropriate constitutive relationships to define how the stress is generated within the ice. This is especially difficult if the material experiences a range of flow regimes, for example, from rapidly flowing to fully stationary, since the constitutive relationships differ between flow regimes. This leads to difficulties modelling behaviour such as segregation and jamming. An alternative approach is to consider the forces applied on each ice floe from the water, riverbanks, and surrounding ice. This approach can be achieved using the DEM. By considering the motion and eventual arrest of each individual ice floe, there is the potential to gather new insight about the formation, evolution, and release of an ice jam.

The primary motivation of the study is to develop a method suitable for simulating smallscale river ice dynamics problems. The study involved two components: first, to enable the accurate simulation of free-surface flows containing solids, resolving both fluid-solid and solidsolid interactions; and second, to apply these capabilities to river ice dynamics problems. The model, however, is not particular to river ice dynamics problems and could be applied to other physical problems involving free-surface flows containing solids.

Section 2 describes the SPH method for the fluid phase, section 3 presents the DEM for the solid phase, and section 4 describes the approach used to couple them. Section 5 describes the results from validation test cases using SPH, DEM, and the coupled SPH-DEM models. Section 6 describes the simulation of two river ice applications. The first consists of a single floating block approaching a stationary cover. The simulation is run at different upstream velocities to determine the critical value for which submergence of the ice block occurs. The second simulation shows accumulation of ice upstream of a set of narrowly spaced piers. 
Robb, D., Gaskin, S.J., Marongiu, J-C (2016) SPH-DEM model for free surface flows containing solids applied to river ice jams, Journal of Hydraulic Research, 54(1): 27-40. doi: 10.1080/00221686.2015.1131203

\section{Smoothed particle hydrodynamics}

SPH is a numerical method for solving fluid dynamics problems, whereby the fluid is represented by a set of particles. A particle can be thought of as a fluid parcel having the same volume of fluid over time or, equivalently, as an interpolation point over which fluid variables, such as velocity, density, and pressure, are calculated. The fluid variables associated with a particle move with it and change with time according to the equations governing the fluid dynamics problem. These fluid variables are interpolated over the values at neighbouring particles using a kernel function.

In the SPH method, a function $f$ representing a fluid variable such as velocity, density, or pressure, associated with a particle $i$ located at a position $\boldsymbol{x}_{\boldsymbol{i}}$ can be written as:

$$
\left\langle f\left(\boldsymbol{x}_{\boldsymbol{i}}\right)\right\rangle=\sum_{j \in D_{i}} f\left(\boldsymbol{x}_{\boldsymbol{j}}\right) W\left(\boldsymbol{x}_{\boldsymbol{i}}-\boldsymbol{x}_{\boldsymbol{j}}, h\right) \omega_{j},
$$

where $W$ is the kernel function depending on a smoothing length $h, \omega_{j}$ is the volume of a neighbouring particle $j$, and $D_{i}$ is the kernel support of particle $i$, i.e., the region over which the interpolation occurs. The angle brackets \langle\rangle indicate that the left-hand side of Eq. (1) is a field approximation, while the function value inside the summation is different. For readability, the angle brackets are omitted for the remainder of the paper. The gradient of $f$ can then be expressed as:

$$
\nabla_{i}\left(f\left(\boldsymbol{x}_{\boldsymbol{i}}\right)\right)=\sum_{j \in D_{i}}\left(f\left(\boldsymbol{x}_{\boldsymbol{j}}\right)-f\left(\boldsymbol{x}_{\boldsymbol{i}}\right)\right) \nabla_{i} W\left(\boldsymbol{x}_{\boldsymbol{i}}-\boldsymbol{x}_{\boldsymbol{j}}, h\right) \omega_{j}
$$

Following the work by Vila (1999), the Euler equations for an inviscid fluid can be solved in the conservative form in an arbitrary moving frame of reference, i.e., in an Arbitrary LagrangianEulerian (ALE) description. Interactions between neighbouring particles are treated as fluxes of mass and momentum. Pairwise numerical fluxes are computed from solutions to one-dimensional moving Riemann problems that can be expressed at the midpoint between the two particles' centres. Compared to the classical SPH approach, SPH-ALE with Riemann solvers does not introduce any explicit artificial viscosity or diffusion term and, thus, does not need additional numerical parameters whose calibration can be tedious. Introduction of Riemann solvers leads to an improved numerical stability and in particular the so-called tensile instability was not observed with this method. An important aspect of SPH-ALE is that it produces smoother pressure fields compared to the classical SPH approach, as can be seen from results in Marongiu, Leboeuf, Favre, and Parkinson (2006) and in Marongiu, Leboeuf, Caro, and Parkinson (2010). Discretizing the continuity and momentum equations in conservative form by the SPH operators above leads to the following SPH-ALE formulation: 


$$
\left\{\begin{array}{l}
\frac{\mathrm{d}}{\mathrm{d} t}\left(\boldsymbol{x}_{\boldsymbol{i}}\right)=\boldsymbol{u}_{\mathbf{0}} \\
\frac{\mathrm{d}}{\mathrm{d} t}\left(\omega_{i}\right)=\omega_{i} \sum_{j \in D_{i}} \omega_{j}\left(\boldsymbol{u}_{\mathbf{0}}\left(\boldsymbol{x}_{\boldsymbol{i}}, t\right)-\boldsymbol{u}_{\mathbf{0}}\left(\boldsymbol{x}_{\boldsymbol{j}}, t\right)\right) \nabla_{i} W_{i j} \\
\frac{\mathrm{d}}{\mathrm{d} t}\left(\omega_{i} \rho_{i}\right)+\omega_{i} \sum_{j \in D_{i}} \omega_{j} 2 \rho_{i j}^{*}\left(\boldsymbol{u}_{\boldsymbol{i j}}^{*}-\boldsymbol{u}_{\mathbf{0}}\left(\boldsymbol{x}_{\boldsymbol{i}}, t\right)\right) \nabla_{i} W_{i j}=0 \\
\frac{\mathrm{d}}{\mathrm{d} t}\left(\omega_{i} \rho_{i} \boldsymbol{u}_{\boldsymbol{i}}\right)+\omega_{i} \sum_{j \in D_{i}} \omega_{j} 2\left[\rho_{i j}^{*} \boldsymbol{u}_{\boldsymbol{i j}}^{*} \otimes\left(\boldsymbol{u}_{\boldsymbol{i j}}^{*}-\boldsymbol{u}_{\mathbf{0}}\left(\boldsymbol{x}_{\boldsymbol{i j}}, t\right)\right)+P_{i j}^{*}\right] \nabla_{i} W_{i j}=\omega_{i} \rho_{i} g+\boldsymbol{F}_{\partial \Omega \rightarrow i}
\end{array}\right.
$$

where the superscript $(*)$ denotes the approximate solution to the Riemann problem at the midpoint between particle pairs, $\boldsymbol{u}_{\mathbf{0}}$ is the particle velocity, $\rho$ is density, $P$ is pressure, $g$ is gravitational acceleration, and $\boldsymbol{x}_{\boldsymbol{i}}$ is the position vector at the midpoint between particle pairs, $i$ and j. $\quad \boldsymbol{F}_{\partial \Omega \rightarrow i}$ is the fluid-solid coupling force exerted on fluid particle $i$ due to a solid boundary $\partial \Omega$ located within the kernel support of $i$. Equation (3) is integrated in time using a third-order explicit scheme. As in most SPH formulations, the equation of state $P=\rho_{0} c_{0}^{2} / \gamma\left[\left(\rho / \rho_{0}\right)^{\gamma}-1\right]$ (Batchelor, 1967) is used to relate density to pressure, where $\rho_{0}$ is the reference density for water $1000 \mathrm{~kg} / \mathrm{m}^{3}, \gamma$ is a constant equal to 7 , and $c_{0}$ is the numerical speed of sound. The selection of the speed of sound as $c_{0}>10 u_{\max }$, where $u_{\max }$ is the maximum velocity in the fluid, makes the water virtually incompressible and limits the density fluctuations to less than $1 \%$ while not requiring a timestep that is prohibitively small.

For hydrodynamic applications, linearized Riemann solvers proved to be sufficient. The Primitive Variable Riemann Solver (PVRS) (Toro, 2009) was adapted for the equation of state in the present model and used for the computations presented herein. Details on this solver and on the adapted second-order MUSCL scheme used in this study are described in Marongiu et al. (2010). Equation (3) makes use of the standard SPH gradient operator. It can be replaced by the renormalized operator introduced by Vila (1999), but the management of this operator for fluid particles close to a solid wall can be difficult due to poor matrix conditioning. Accordingly, it was decided for this study, where the coupling between fluid and solid motion is important, not to use the renormalized operator.

A Riemann problem is composed of a left and right state separated by an interface. Here, the left and right states are located at SPH fluid particles and the interface is the midpoint between them. For the interaction between a fluid particle and a boundary, a boundary flux can be computed from the solution to the Riemann problem between the fluid particle and the boundary. It is a one-dimensional partial Riemann problem in the direction normal to the boundary surface. It is a partial Riemann problem because one state, on the boundary surface, is missing but the imposed physical boundary condition provides a part of the solution. Dubois (2001) developed partial Riemann solvers to solve the interaction between a fluid state and the imposed physical boundary condition. Adaptation of this approach to the equation of state used in this study is described further in Marongiu et al. (2010). 


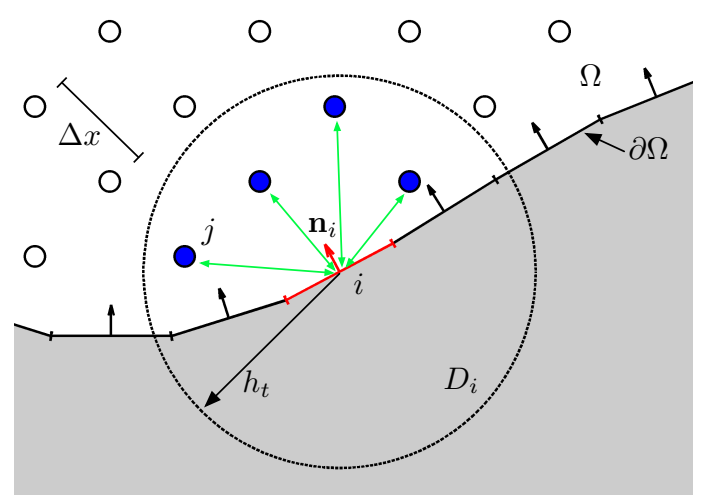

Figure 1 Boundary treatment definition sketch: boundary surface element $i$ (red) with unit normal vector $\boldsymbol{n}_{\boldsymbol{i}}$, and kernel support $D_{i}$. The interpolation is over the fluid particles $j$ (blue) in $D_{i}$. The green arrows represent boundary fluxes.

The treatment of boundary conditions in SPH-ALE is then two-fold. The boundary surface is first discretized into surface elements whose size is similar to the size of the fluid particles. A surface element $i$ is defined by its centre $\boldsymbol{x}_{\boldsymbol{i}}$, the unit vector normal to the surface at its centre $\boldsymbol{n}_{\boldsymbol{i}}$, and its surface area $\omega_{i}$. A surface element located in the kernel support of a neighbouring fluid particle is included in the SPH interpolation and contributes to the acceleration of the fluid particle through boundary fluxes. Conversely, a fluid particle located in the kernel support of a neighbouring surface element is included in the SPH interpolation and contributes to the pressure exerted on the surface element by the fluid. Figure 1 shows a graphical representation of the SPH-ALE boundary treatment. The computation of boundary contributions is described further in Marongiu et al. (2010), and Leboeuf and Marongiu (2011).

From the computation of boundary fluxes between boundary surface elements and fluid particles, it is possible to compute the resulting force and torque exerted by the fluid on a solid. The pressure on a surface element $i$ is given by:

$$
P_{i}=\sum_{j \in D_{i}} 2 \omega_{j} P_{i j}^{*} W_{i j}
$$

To calculate the force exerted by the fluid in the fluid domain $\Omega$ on a solid boundary $\partial \Omega, \boldsymbol{F}_{\Omega \rightarrow \partial \Omega}$, the pressure on each surface element $i$ belonging to a given solid can be integrated over the surface of the solid, which gives:

$$
\boldsymbol{F}_{\Omega \rightarrow \partial \Omega}=-\sum_{i \in \partial \Omega} \omega_{i} P_{i} \boldsymbol{n}_{\boldsymbol{i}}
$$

Recall that one of the objectives of the study is to enable the accurate simulation of free-surface flows containing solids. The ALE description presented herein makes the boundary treatment applicable to both stationary and moving solid boundaries. For stationary boundaries, the particle velocity $\boldsymbol{u}_{\mathbf{0}}$ is equal to 0 . For moving boundaries, the value of $\boldsymbol{u}_{\mathbf{0}}$ changes with time according to the forces exerted on the solid by the fluid or by other solids. 
Robb, D., Gaskin, S.J., Marongiu, J-C (2016) SPH-DEM model for free surface flows containing solids applied to river ice jams, Journal of Hydraulic Research, 54(1): 27-40. doi: 10.1080/00221686.2015.1131203

\section{Discrete element method}

The DEM is used for solving problems involving the dynamics of granular systems, whereby the trajectories of each particle are calculated from the collisions and contacts with neighbouring particles. In this work, the DEM is divided into two types: hard-sphere and soft-sphere models (Van der Hoef et al., 2006). The hard-sphere model has been implemented to treat solid bodies of arbitrary shape and the soft-sphere model can simulate circular solids in two dimensions and spherical solids in three dimensions.

In the hard-sphere model, the particles follow piece-wise linear trajectories between inelastic collisions. The interactions between particles are assumed to be instantaneous, where momentum is exchanged by impulsive forces. The dissipation of energy from the inelastic collisions is quantified by a coefficient of restitution, specifying the ratio of recoil velocity to approach velocity. For lower density systems, the hard-sphere model is less computationally demanding than the soft-sphere model. For closely packed particles or low coefficients of restitution, the collisions can lead to large decreases in kinetic energy, such that the relative velocities of colliding particles approach zero. In this limit, the particles tend to be in resting contact with their neighbours. The hard-sphere model depends on the assumption that particles experience instantaneous collisions. For particles in resting contact, this assumption is violated and the hard-sphere model is no longer valid. The hard-sphere model used in this study can treat objects of any arbitrary shape by considering solid collisions with instantaneous exchanges of momentum and applying the principles of rigid body dynamics.

In the soft-sphere model, particles are allowed to overlap slightly and the forces are calculated through a contact force relationship. The particle-particle contacts can last extended durations and, in dense systems, force networks can develop between clusters of contacting particles. The soft-sphere model can successfully simulate slowly moving and static granular systems, where the hard-sphere model breaks down. This is achieved at the cost of a longer computation time. The contact forces are calculated based on the amount of overlap between particles, which depends on the particles' material properties. These are commonly described in terms of a spring stiffness, friction angle, and damping constant. The soft-sphere model used in this study is a simple and robust linear spring and dashpot model (Cundall \& Strack, 1979). The model has been implemented to simulate circular solids in two dimensions and spherical solids in three dimensions. Figure 2a shows a schematic of two solids in contact with each other and Fig. $2 \mathrm{~b}$ shows a graphical representation of a spring and dashpot system.

In the linear spring and dashpot system, the normal force $\boldsymbol{F}_{\boldsymbol{n}}$ on solid $a$ by solid $b$ can be calculated by:

$$
\boldsymbol{F}_{\boldsymbol{n}}=-k_{n} \delta_{n} \cdot \boldsymbol{n}_{\boldsymbol{a b}}-\eta_{n} \boldsymbol{v}_{\boldsymbol{n}}
$$

where $k_{n}$ is the normal spring stiffness, $\boldsymbol{n}_{a b}$ is the unit vector pointing from the centre of mass of solid $a$ to the centre of mass of solid $b, \eta_{n}$ is the normal damping coefficient, and $\boldsymbol{v}_{\boldsymbol{n}}$ is the normal relative velocity. The magnitude of the tangential force is: 

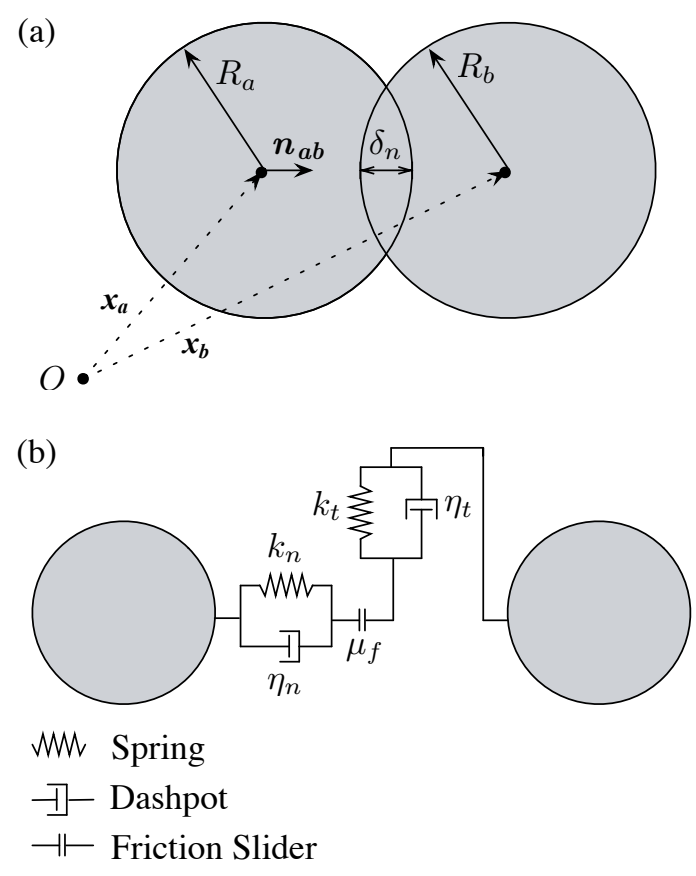

Figure 2 Soft-sphere model: (a) nomenclature and (b) spring and dashpot system

$$
F_{t}=\min \left\{\mu\left|\boldsymbol{F}_{\boldsymbol{n}}\right|,\left|k_{t} \int \boldsymbol{v}_{\boldsymbol{t}} \mathrm{d} t+\eta_{t} \boldsymbol{v}_{\boldsymbol{t}}\right|\right\}
$$

where $\mu$ is the coefficient of friction related to the internal angle of friction $\phi$ by $\mu=\tan \phi$, and $v_{t}$ is the relative tangential velocity. The damping coefficient dictates the amount of energy lost during a collision, that is, the inelasticity of the material. It can be related to the coefficient of restitution $\varepsilon$ by:

$$
\eta_{n}=-\frac{2 \ln (\varepsilon) \sqrt{m_{\mathrm{eff}} k_{n}}}{\sqrt{\pi^{2}+\ln ^{2}(\varepsilon)}}
$$

where $m_{\mathrm{eff}}=1 / m_{a}+1 / m_{b}$. To produce accurate results, the contact force relationships should be evaluated over more than 40 or 50 timesteps (Walton, 1993). The typical contact duration $t_{c}$ can be expressed as:

$$
t_{c}=\sqrt{\frac{\pi^{2}+\ln ^{2}(\varepsilon)}{k_{n} / m_{\mathrm{eff}}}} ;
$$

therefore, the timestep is $\Delta t=t_{c} / 50$. Although $k_{n}$ can be related to the physical properties of the simulated material, for example, Young's modulus, this value often produces prohibitively small timesteps. Instead, $k_{n}$ is chosen so that the contact time remains over 50 timesteps while the maximum overlap $\delta_{n}$ is less that $1 \%$ of the particle diameter. The timestep requirement for the soft-sphere model is generally smaller than the timestep requirement for the SPH method. Recall that in the hard-sphere model, collisions are instantaneous; i.e. they occur over a single timestep. Therefore, the restriction $\Delta t=t_{c} / 50$ does not apply, allowing for faster computations. 
To ensure the stability of a coupled SPH-DEM model, the minimum timestep of the two methods sets the timestep for the coupled model.

Both the hard-sphere and the soft sphere models are used in this study. The hard-sphere model is used to investigate the stability of a floating ice block approaching a stationary cover and the soft-sphere model is used to simulate the accumulation of ice upstream of an obstruction.

\section{Fluid-solid coupling}

The numerical results presented in this paper show simulations of free-surface fluid flows containing solids. The motion of a solid is governed by the force exerted by the fluid, by any contacts forces with other solids, and by gravitational forces.The fluid-solid and solid-fluid interactions are calculated using a partial Riemann solver described in section 2. The pressure on each surface element of a solid is calculated and integrated over the solid's surface area to determine the force on the solid by the fluid. The surface elements are included in the SPH interpolation of neighbouring fluid particles, resulting in a calculation of the force exerted by the solid on the fluid. The solid-solid interactions are calculated using either the hard-sphere model or the soft-sphere model, as described in section 3 .

The forces acting on a solid can be divided into contact forces, hydraulic forces and gravitational forces. Given a solid object $a$, the equations describing its motion are:

$$
\begin{gathered}
m_{a} \frac{\mathrm{d}^{2} \boldsymbol{x}_{\boldsymbol{a}}}{\mathrm{d} t^{2}}=\boldsymbol{F}_{\text {solid } \rightarrow a}+\boldsymbol{F}_{\text {fluid } \rightarrow a}+m_{a} g, \\
\mathrm{l}_{\mathrm{a}} \frac{\mathrm{d} \boldsymbol{\omega}_{\boldsymbol{a}}}{\mathrm{d} t}=\sum_{i \in a}\left(\boldsymbol{p}_{\boldsymbol{i}}-\boldsymbol{x}_{\boldsymbol{a}}\right) \times \boldsymbol{F}_{\boldsymbol{i}},
\end{gathered}
$$

where $m_{a}$ is the mass of solid $a, \boldsymbol{x}_{\boldsymbol{a}}$ is the centre of mass of the solid, $\boldsymbol{F}_{\text {solid } \rightarrow a}$ includes the forces on $a$ from any solids in contact with $a, \boldsymbol{F}_{\text {fluid } \rightarrow a}$ includes the hydraulic forces calculated on the surface elements $i$ associated with solid $a$ (equation 5), $\mathrm{I}_{\mathrm{a}}$ is the moment of inertia, $\omega_{a}$ is the rotational velocity, $\boldsymbol{p}_{\boldsymbol{i}}$ is the position of the $i^{\text {th }}$ surface element on the solid surface, $\boldsymbol{F}_{\boldsymbol{i}}$ is any surface force including contact forces and hydraulic forces acting on solid $a$ at $\boldsymbol{p}_{\boldsymbol{i}}$, and the vector product is denoted by the symbol $\times$. Equations (10) and (11) are integrated in time to determine the translational and rotational velocity of the solid for the next timestep. Since the velocity of a surface element is given by:

$$
\frac{\mathrm{d} \boldsymbol{p}_{i}}{\mathrm{~d} t}=\boldsymbol{v}_{\boldsymbol{a}}+\boldsymbol{\omega}_{\boldsymbol{a}} \times\left(\boldsymbol{p}_{\boldsymbol{i}}-\boldsymbol{x}_{\boldsymbol{a}}\right)
$$

then equation (12) can be integrated in time to determine the new position of each boundary element making up the solid's surface. This approach conserves both linear and angular momentum (Monaghan et al., 2003). 
Robb, D., Gaskin, S.J., Marongiu, J-C (2016) SPH-DEM model for free surface flows containing solids applied to river ice jams, Journal of Hydraulic Research, 54(1): 27-40. doi: 10.1080/00221686.2015.1131203

\section{Application to free-surface flows containing solids}

In this section, different test cases are presented to verify that the proposed SPH-DEM model accurately simulates both fluid-solid and the solid-solid interactions. Initially, simulations are run without a fluid phase so that the particle interactions of the solids can be observed without the effect of hydraulic forces. Afterwards, a fluid is introduced to evaluate the motion of the solids due to interactions with both the fluid and other solids.

Each DEM technique is evaluated separately with simulations that test their respective contact force schemes. Since the hard-sphere model is based on instantaneous exchanges of momentum between colliding solids, the validation is made through a comparison of the computational results to a simple analytical solution for the rebound height of a solid dropped onto a level surface.

Compared to the hard-sphere model, the soft-sphere model can be applied to a broader range of flow regimes, from rapid granular flow to granular material in static equilibrium. As a result, the validation test case was chosen so that the granular material experiences this range of flow regimes. In the test case, the granular assembly initially deforms under the force of gravity onto a flat surface to form a pile. The granular material is initially rapidly flowing and finishes in static equilibrium, where the particles form a force network that transmits its bulk weight onto the supporting surface. The computational results are compared to the expected value of the bulk weight of granular material that is transmitted from the particles onto the supporting surface. The final height and the final horizontal distance of the pile are compared to a scaling law based on laboratory experiments of the collapse of granular columns.

After the DEM techniques are evaluated in the absence of hydraulic forces, a fluid is added to the simulation to assess the solid motion due to the fluid flow. The effects of dynamic forces, hydrostatic forces, and buoyancy are tested. The computational results are compared to previously published laboratory experiments and analytical solutions.

\subsection{Hard-sphere model-bouncing sphere}

The hard-sphere model is based on instantaneous exchanges of momentum between colliding solids. To validate this model, a single sphere is dropped onto a level surface and rebounds to a given height according to its coefficient of restitution. The sphere is allowed to rebound many times to observe the successive decreases in rebound height due to dissipation of energy from the collision with the surface. The problem domain contains one sphere of radius $0.05 \mathrm{~m}$ whose bottom is $0.5 \mathrm{~m}$ above a level flat surface as shown in Fig. 3. The sphere is dropped and falls under the force of gravity until it contacts the level surface. It then rebounds to a maximum height less than the original height according to its coefficient of restitution of 0.8 . The height of the solid over time is compared to the analytical solution in Fig. 4.

The rebound height and timing agrees very well with the analytical solution. The computational results only start to deviate from the analytical solution once the rebound height is roughly the same size as the discretization size $\Delta x=0.005 \mathrm{~m}$ of the sphere and the level surface. It is, 
Robb, D., Gaskin, S.J., Marongiu, J-C (2016) SPH-DEM model for free surface flows containing solids applied to river ice jams, Journal of Hydraulic Research, 54(1): 27-40. doi: 10.1080/00221686.2015.1131203

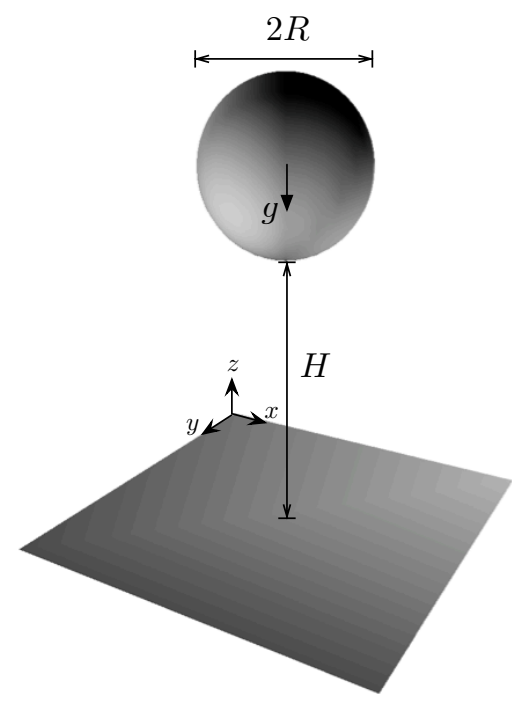

Figure 3 Solid dropping onto a level surface: definition sketch

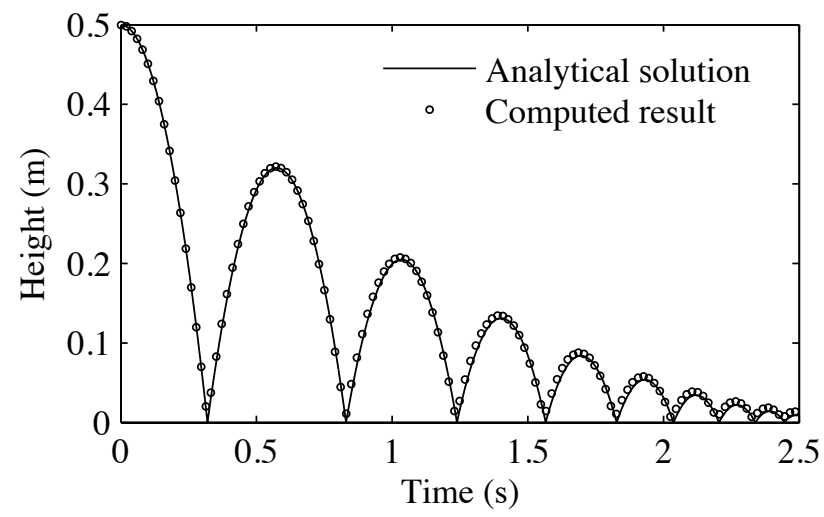

Figure 4 Solid dropping onto a level surface: numerical results compared to the analytical solution 
Robb, D., Gaskin, S.J., Marongiu, J-C (2016) SPH-DEM model for free surface flows containing solids applied to river ice jams, Journal of Hydraulic Research, 54(1): 27-40. doi: 10.1080/00221686.2015.1131203
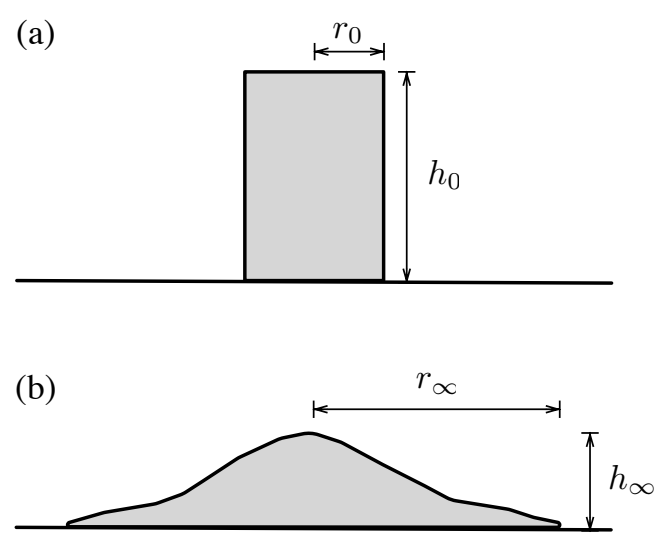

Figure 5 Granular collapse: definition sketch

therefore, understandable that there are small differences from the analytical solution for these small rebound heights.

\subsection{Soft-sphere model - granular collapse in two dimensions}

The collapse of a granular column onto a horizontal plane is simulated in two dimensions. The purpose of the simulation is (1) to observe the range of granular flow regimes, from rapidly flowing to static, and (2) to determine, once the flow comes to rest and forms a pile, if the force network between particles accurately transmits the bulk weight of the pile onto the horizontal plane. The height of the final deposit and the run-out are compared with the scaling laws proposed by Lajeunesse, Mangeney-Castelnau, and Vilotte (2004) who carried out laboratory experiments of granular collapses.

In this simulation, a square two-dimensional granular column is released from rest and forms a pile. The aspect ratio $a=h_{0} / r_{0}$ of the column is equal to 2 and the internal angle of friction of the granular material is $20^{\circ}$. The two-dimensional computational domain contains a granular column of length $2.5 \mathrm{~m}$ and height $2.5 \mathrm{~m}$ and a total of 1024 solids. Each solid has a density $\rho_{s}=1000 \mathrm{~kg} / \mathrm{m}^{3}$, weighing $2.83 \mathrm{~kg}$ per metre depth. The solid has a normal spring stiffness of $10^{5} \mathrm{~N} / \mathrm{m}$ and a coefficient of restitution of 0.8. A problem definition sketch is shown in Fig. 5 with $r_{0}=1.25 \mathrm{~m}$ and $h_{0}=2.50 \mathrm{~m}$.

Figure 6 shows a time sequence of the granular collapse. The vertical force exerted on the horizontal surface is plotted in Fig. 7. After the pile reaches static equilibrium, the vertical force on the surface should equal the bulk weight of the granular material indicated in Fig. 7 by the dashed line. The force time history in Fig. 7 shows that the pile approaches static equilibrium near the end of the simulation and the force calculated on the supporting surface agrees with the bulk weight of the granular material.

The collapse of a granular column occurs in several stages: (1) a free-fall regime as the intial potential energy is converted into kinetic energy, (2) a heap-flow regime as the vertical kinetic energy is converted into horizontal kinetic energy and (3) the shallow layer regime in which the 
Robb, D., Gaskin, S.J., Marongiu, J-C (2016) SPH-DEM model for free surface flows containing solids applied to river ice jams, Journal of Hydraulic Research, 54(1): 27-40. doi: 10.1080/00221686.2015.1131203
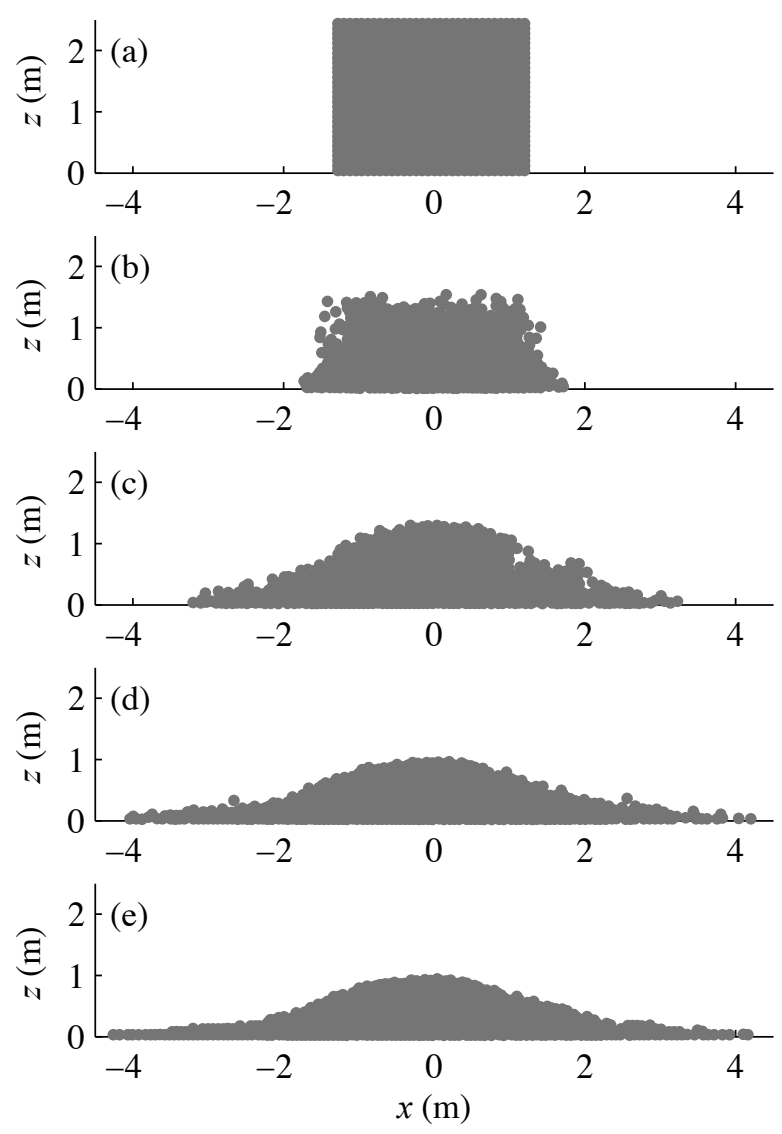

Figure 6 Granular collapse: time sequence showing a granular collapse for times (a) $0 \mathrm{~s}$, (b) $0.5 \mathrm{~s},(\mathrm{c}) 1.0 \mathrm{~s}(\mathrm{~d}) 1.5 \mathrm{~s}$, and (e) $2.0 \mathrm{~s}$

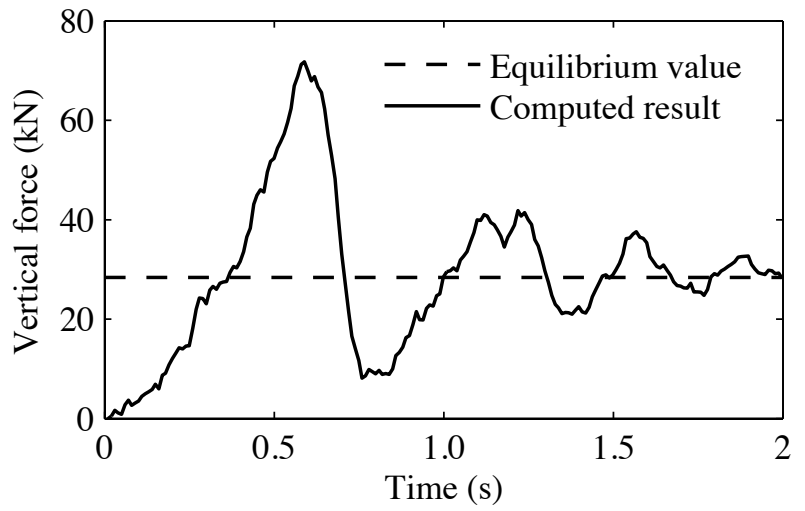

Figure 7 Granular collapse: vertical force exerted on the horizontal plate compared to the bulk weight of the pile in static equilibrium 
Robb, D., Gaskin, S.J., Marongiu, J-C (2016) SPH-DEM model for free surface flows containing solids applied to river ice jams, Journal of Hydraulic Research, 54(1): 27-40. doi: 10.1080/00221686.2015.1131203

horizontal kinetic energy is eventually dissipated through particle interactions (Staron \& Hinch, 2005; Lacaze \& Kerswell, 2009). The unsteady and non-uniform dynamics of the spreading of the granular collumn are due to the particle interactions and result in the oscillations in the observed vertical force on the plate. The first peak represents the impact of the free-fall stage and the subsequent oscillations are interpreted as due to the combined effect of the bouncing and the particle-particle interactions.

Lajeunesse et al. (2004) proposed that for aspect ratios $a$ greater than 0.74 , the run-out distance $r_{\infty}$ is related to $a$ by $\left(r_{\infty}-r_{0}\right) / r_{0}=2 a^{0.5}$ and the deposition height is $h_{\infty} / r_{0}=0.74$. The scaling law predicts $r_{\infty}=4.8 \mathrm{~m}$ and $h_{\infty}=0.93 \mathrm{~m}$. The simulated results agree reasonably well, showing $r_{\infty}=4.3 \mathrm{~m}$ and $h_{\infty}=0.93 \mathrm{~m}$.

From this test case, the following conclusions and observations can be made: (1) the computational results show some of the key characteristics of a granular collapse, (2) the bulk weight of the granular material is accurately calculated for a pile in static equilibrium through a network of contact forces, and (3) the run-out length and depositional height agree reasonably well with the scaling law proposed by Lajeunesse et al. (2004).

\subsection{Wedge entry}

The purpose of this test case is to evaluate the fluid-solid interactions in the proposed model. The simulation involves the free fall of a triangular wedge entering hydrostatic water. The hydraulic force time history is compared to laboratory experiments conducted by Zhao, Faltinsen, and Aarsnes (1997) and numerical experiments using SPH by Oger, Doring, Alessandrini, and Ferrant (2006).

The laboratory experiments in Zhao et al. (1997) involved a triangular wedge attached to a trolley with vertical guide-rails that restrict any horizontal or angular motion. The wedge is raised to a specified height above the hydrostatic water, and then released from rest. The force, pressure, acceleration and velocity time histories are measured using instrumentation detailed in their paper. The numerical simulation presented herein allows the wedge to move freely in the vertical direction subject to gravitational and hydrodynamic forces, but its horizontal motion and rotation are restricted to mimic the test rig used in the experiment.

The two-dimensional computational domain has one symmetrical triangular wedge of width $0.5 \mathrm{~m}$ with a $30^{\circ}$ deadrise angle. The tank is $4 \mathrm{~m}$ wide, $2 \mathrm{~m}$ tall, and contains still water with a hydrostatic pressure distribution. In Zhao's experiment, the wedge is dropped from $2 \mathrm{~m}$ above the free surface to reach a velocity of $6.15 \mathrm{~m} / \mathrm{s}$ just before entry. For the numerical simulation presented herein, the wedge is placed just above the free surface and given an initial velocity of $6.15 \mathrm{~m} / \mathrm{s}$. This eliminates the needless computation time of the wedge falling solely under the force of gravity. Figure 8 shows the geometry of the system.

Figure 9 compares the vertical force history on the wedge calculated in the present study $(\Delta x=0.002 \mathrm{~m})$ to the experimental results in Zhao et al. (1997) and the numerical results in Oger et al. (2006). The SPH force calculations agree well with the experiments in Zhao et al. 


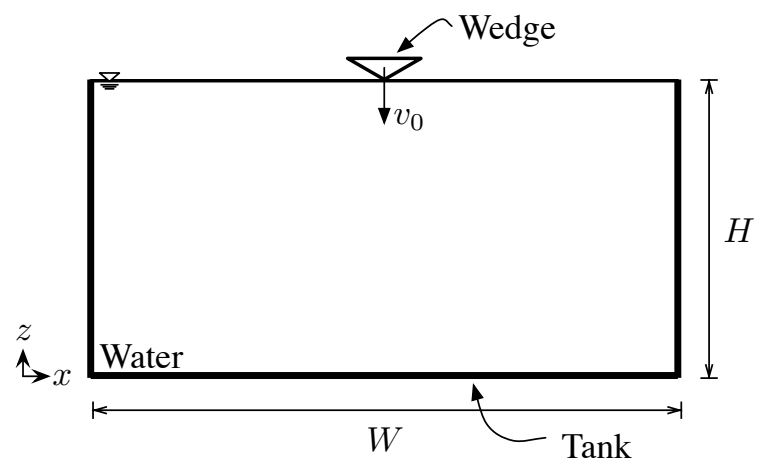

Figure 8 Wedge entry: definition sketch

(1997). In the first $0.0025 \mathrm{~s}$, the SPH results in the present study oscillate noticeably and over predict the expected values of vertical force on the wedge. This error may be due to using a discretization size that is too coarse. Recall that a solid is composed of boundary elements that are used to calculate the pressure on the solid. As the wedge enters the water, there are initially very few boundary elements in contact with the water. This could lead to jumps and drops in the force calculation as observed in Fig. 9. Oger et al. (2006) reduce this error by using a variable particle resolution with fine particles $(\Delta x=0.00077 \mathrm{~m})$ in the region where the wedge penetrates the free surface. After $0.0025 \mathrm{~s}$, the force history becomes smoother and closely follows the experimental results until the force on the wedge starts to decrease. For the remainder of the simulation, the computed force over estimates the experimental values. The overestimation may be due to three-dimensional effects that are not taken into account in the numerical simulation.

The relative $L_{2}$ norm is used to perform an error analysis and to examine the influence of spatial resolution for simulations conducted with initial particle spacing $\Delta x=0.01,0.005,0.002$, and $0.001 \mathrm{~m}$. The relative $L_{2}$ norm of the error $\varepsilon_{L 2}$ is given by:

$$
\varepsilon_{L_{2}}=\sqrt{\frac{\sum_{i=1}^{N}\left(\phi_{e, i}-\phi_{n, i}\right)^{2}}{\sum_{i=1}^{N}\left(\phi_{e, i}\right)^{2}}},
$$

where $\phi$ is a general variable, $N$ is the number of sample points, and the subscripts $e$ and $n$ refer to the experimental and numerical solutions, respectively. Figure 10 shows the relative $L_{2}$ norm error in the vertical force exerted on the wedge compared to the experimental results in Zhao et al. (1997). This figure shows the results converging to a solution close to the experimental results in Zhao et al. (1997) at a rate of 0.75 for the three coarsest particle resolutions. The convergence rate decreases with further decrease in $\Delta x$ indicating convergence to a minimum error $\varepsilon_{L_{2}}=10^{-1}$ and thus particle resolution independence.

\subsection{Falling solids with different densities}

The purpose of the simulation of falling solids with different densities is to evaluate the effects of buoyancy on a solid in a free-surface flow. The simulation involves the free fall of a solid 
Robb, D., Gaskin, S.J., Marongiu, J-C (2016) SPH-DEM model for free surface flows containing solids applied to river ice jams, Journal of Hydraulic Research, 54(1): 27-40. doi: 10.1080/00221686.2015.1131203

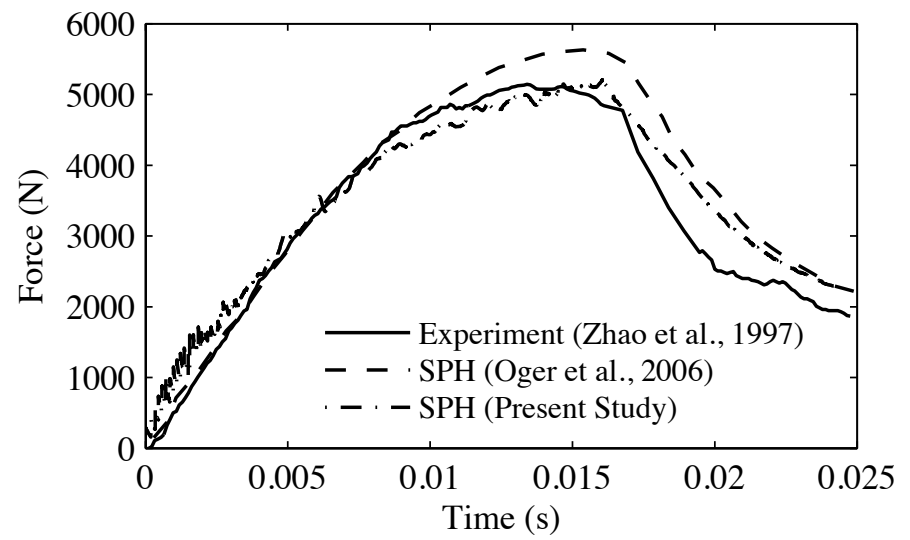

Figure 9 Wedge entry: vertical force time history of the numerical results compared to experiments by Zhao et al. (1997)

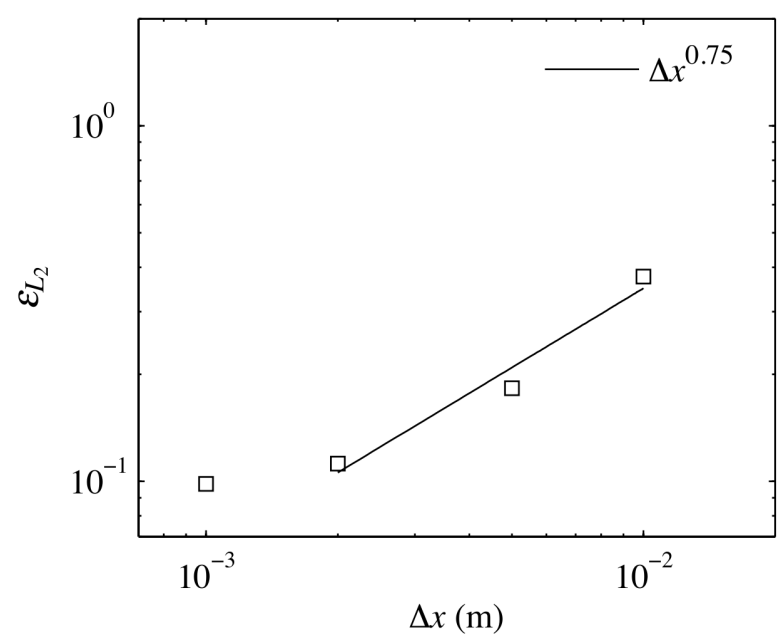

Figure 10 Wedge entry: relative $L_{2}$ norm of the error in vertical force exerted on the wedge for initial particle spacing $\Delta x=0.01,0.005,0.002$, and $0.001 \mathrm{~m}$ 
Robb, D., Gaskin, S.J., Marongiu, J-C (2016) SPH-DEM model for free surface flows containing solids applied to river ice jams, Journal of Hydraulic Research, 54(1): 27-40. doi: 10.1080/00221686.2015.1131203

into still water. The density of the solid is changed from one simulation to the next to observe the effects of buoyancy. The computational results show the same simulation using three different solid densities: 500,1000 , and $2000 \mathrm{~kg} / \mathrm{m}^{3}$. The two-dimensional computational domain contains a solid square of side length $0.1 \mathrm{~m}$ above a water column of width $0.5 \mathrm{~m}$ and height $0.5 \mathrm{~m}$ with an initial hydrostatic pressure distribution. The solid is released from rest under the force of gravity and impacts the free surface. The two-dimensional solid has all its degrees of freedom; i.e., it can move in the $x$ - and $z$-directions as well as rotate about the $y$-axis. The simulations were conducted with initial particle spacing $\Delta x=0.01,0.005,0.0025$, and $0.00125 \mathrm{~m}$, with particle resolution independence occurring at $0.0025 \mathrm{~m}$.

Figure 11 shows the geometry of the system, following the numerical experiments of Lee et al. (2010). Figure 12 shows a time sequence of our numerical simulations. The position of the falling solid computed by Lee et al. (2010) is superimposed onto Figure 12 for comparison with the results from the present study. For the lighter solid, the free surface is disturbed slightly as the solid enters the water. The fluid motions are small due to the disturbance of the solid. As expected, the solid floats with half its volume above the free surface. Observe that the solid rotates $45^{\circ}$. A righting moment analysis shows that at this solid density, this is its natural equilibrium position. The neutrally buoyant solid enters the free surface so that its entire volume is immersed in the water. The heavier solid, as expected, sinks to the bottom and causes the largest fluid motion compared to the lighter solids. The computational results are in agreement with Lee et al. (2010), however small differences in the exact position and orientation of the solid occur for the solid densities of $500 \mathrm{~kg} / \mathrm{m}^{3}$ and $1000 \mathrm{~kg} / \mathrm{m}^{3}$ and, for the solid with a density of $2000 \mathrm{~kg} / \mathrm{m}^{3}$, the solid falls more slowly at $t=0.2,0.4$, and $0.6 \mathrm{~s}$. No detailed experimental data exist for comparison, however the results are in agreement with the expected outcomes following Archimedes' principle.

The simulation of free-surface flows containing solids has been demonstrated. The applicability of these new developments is not exclusive to river ice dynamics, but could be used for many problems including floating debris, landslides, and the movement of rock armour breakwaters. The next section applies the model to two river ice dynamics problems.

\section{River ice dynamics simulations}

The numerical methods described in sections 2 to 4 are applied to two different river ice problems. First, a floating block is simulated approaching a stationary cover. Depending on the block geometry and the flow conditions, it accumulates at the leading edge of the stationary cover or it submerges under the cover. The second simulation involves ice floes accumulating upstream of an obstruction composed of cylindrical piers. This simulation lays the groundwork for more quantitative analyses, where the force on a pier can be estimated taking into account the ice accumulation as well as the hydraulic forces from the fluid flow. 
Robb, D., Gaskin, S.J., Marongiu, J-C (2016) SPH-DEM model for free surface flows containing solids applied to river ice jams, Journal of Hydraulic Research, 54(1): 27-40. doi: 10.1080/00221686.2015.1131203

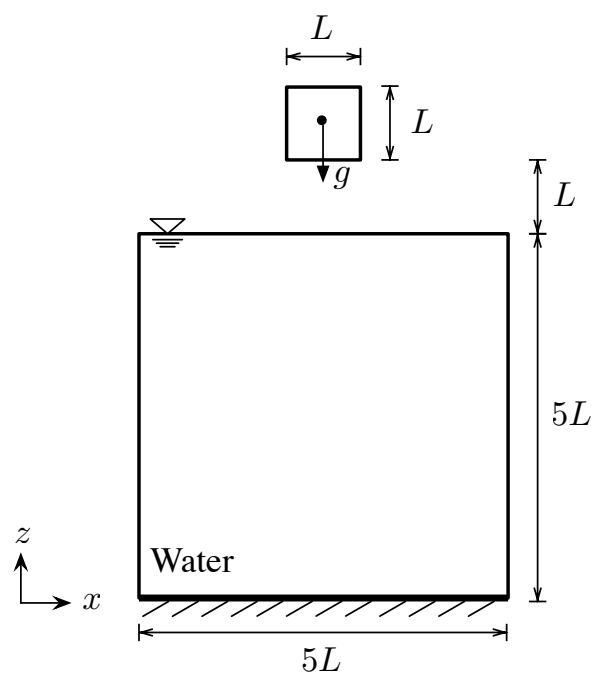

Figure 11 Falling solid with different densities: definition sketch

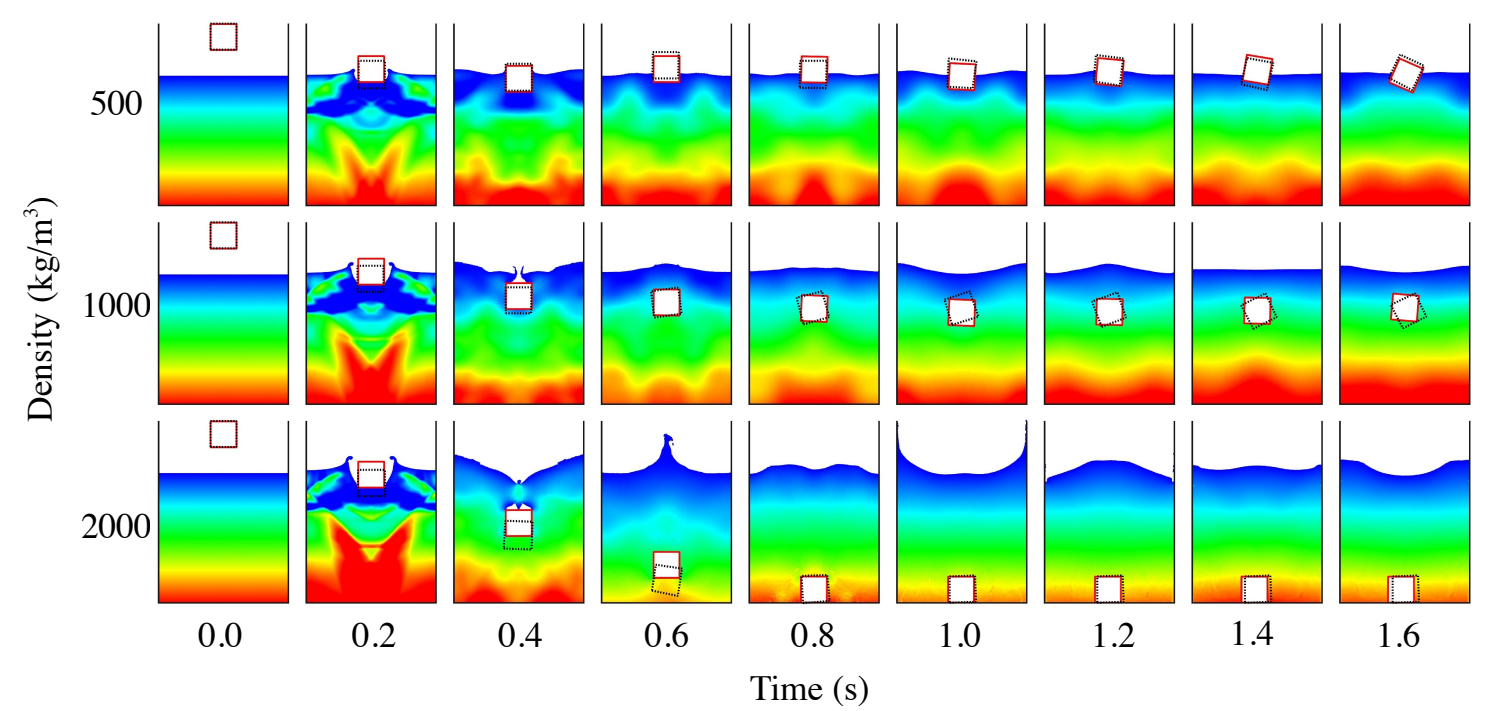

Figure 12 Falling solids with different densities: numerical results. The position of the falling solid in the present study (in red) is compared to the results from Lee et al. (2010) (in black). The colour gradient shows pressure from $0 \mathrm{kPa}$ (blue) to $5 \mathrm{kPa}$ (red). 
Robb, D., Gaskin, S.J., Marongiu, J-C (2016) SPH-DEM model for free surface flows containing solids applied to river ice jams, Journal of Hydraulic Research, 54(1): 27-40. doi: 10.1080/00221686.2015.1131203

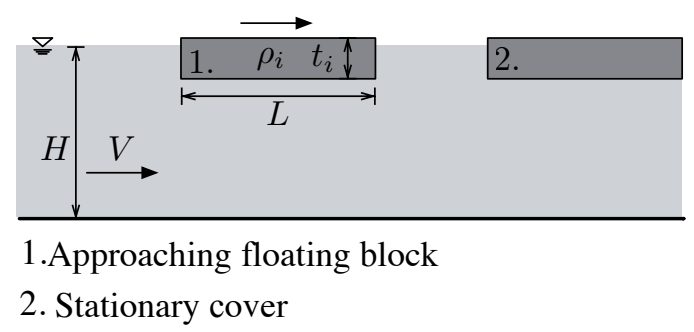

Figure 13 Floating block stability: definition sketch

\subsection{Floating block stability}

To understand the mechanisms behind ice floe accumulation, it is useful to determine under which flow conditions ice floes accumulate and under which conditions they submerge under a stationary cover. Neglecting the viscosity of water and assuming ice and water of constant density, dimensional analysis suggests that the critical upstream velocity $V_{c r}$, for which submergence occurs is a function of gravitational acceleration $g$, upstream water depth $H$, block thickness $t_{i}$, block length $L$, fluid density $\rho_{w}$ and solid density $\rho_{i}$. The relation can be nondimensionalized and expressed in terms of a densimetric Froude number $F_{D}$, based on the depth-averaged velocity $V$ upstream of block 1 in Fig. 13, the block thickness, and density differences:

$$
\mathrm{F}_{\mathrm{D}}=\frac{V}{\sqrt{g t_{i}\left(1-\rho_{i} / \rho_{w}\right)}}=f\left(\frac{t_{i}}{H}, \frac{t_{i}}{L}\right)
$$

A floating block moving at the depth-averaged flow velocity $V$ was simulated approaching a stationary cover. The flow velocity was increased incrementally, corresponding to a range of densimetric Froude numbers between 0.2 and 2 in increments of 0.2 . The block thickness to flow depth ratio $t_{i} / H$ was varied from 0.1 to 0.6 in increments of 0.1 . All other parameters were kept the same. The results were compared to approximate solutions from Pariset and Hausser (1961), Ashton (1974), Daly and Axelson (1990), and with experimental data from Uzuner and Kennedy (1972).

The numerical setup is shown in Fig. 13. The computational domain contains one freely moving block, moving at velocity $V$, of height $0.097 \mathrm{~m}$ and length $0.382 \mathrm{~m}$, and one stationary block of the same length. The solids have a coefficient of restitution of 0.8 , and a density of $874 \mathrm{~kg} / \mathrm{m}^{3}$. The density and geometry in this simulation were chosen to match laboratory experiments by Uzuner and Kennedy (1972). The simulation was conducted with initial particle spacing $\Delta x=0.0085 \mathrm{~m}$.

Figure 14 shows a representative time sequence of a floating block under-turning with a densimetric Froude number greater than the critical value. In total, there were 60 simulations run at different values of $\mathrm{F}_{\mathrm{D}}$ and $t_{i} / H$. Figure 15 compares the results in all of the simulations to approximate solutions and laboratory data. The top limit of the error bar indicates the densimetric Froude number at which under-turning was observed. The bottom limit indicates the nearest simulation at a lower densimetric Froude number at which there was no under-turning. 
Robb, D., Gaskin, S.J., Marongiu, J-C (2016) SPH-DEM model for free surface flows containing solids applied to river ice jams, Journal of Hydraulic Research, 54(1): 27-40. doi: 10.1080/00221686.2015.1131203

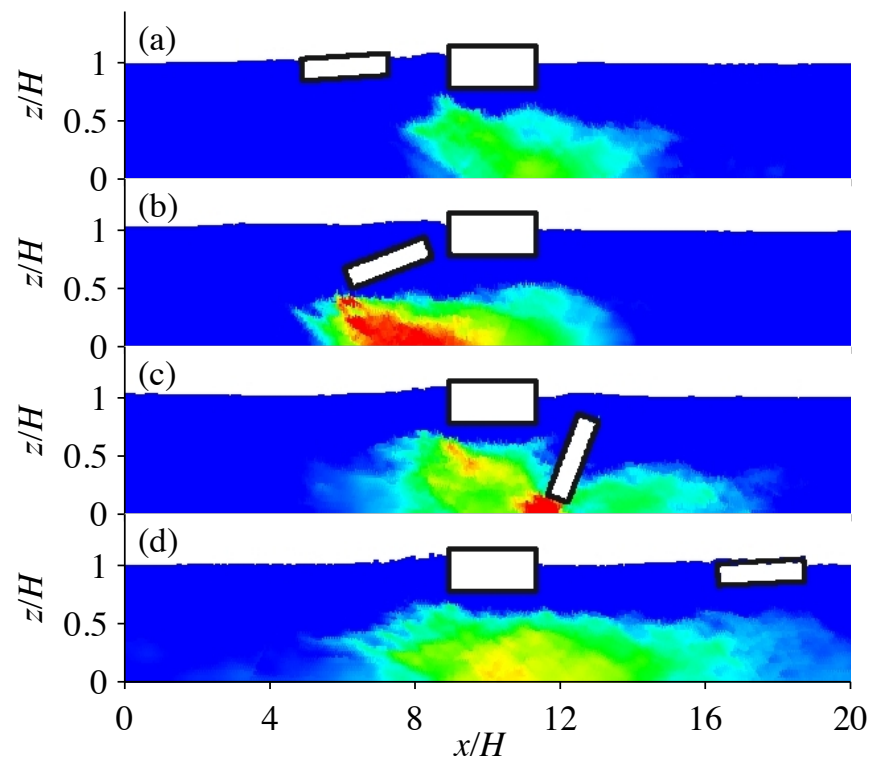

Figure 14 Floating block stability: time sequence of a floating block under-turning with $F_{D}$ greater than the critical value for times (a) $0 \mathrm{~s}$, (b) $2.5 \mathrm{~s}$, (c) $5.0 \mathrm{~s}$, (d) $7.0 \mathrm{~s}$. The colour gradient shows velocity from $0.5 \mathrm{~m} / \mathrm{s}$ (blue) to $0.9 \mathrm{~m} / \mathrm{s}$ (red).

The critical value is, therefore, between these two. From Fig. 15, the simulations agree well with theory and experiments for large values of $t_{i} / H$, from 0.4 to 0.6 . For smaller values of $t_{i} / H$, from 0.1 to 0.3 , the simulations tend to overestimate the critical densimetric Froude number for under-turning.

As the block thickness to flow depth ratio decreases, the resulting pressure distribution under the blocks is dominated by the hydraulics at their leading edge rather than the flow acceleration due to the reduction in cross-section of the flow beneath the blocks. The discrepancies between the computed and the experimental results at low thickness to depth ratios may be due to the model not adequately capturing the important hydraulics occurring at the leading edge of the blocks. This problem is not unlike the flow over a forward facing step where the flow detaches from the upstream corner and reattaches on the block farther downstream, creating a region of increased drag at the leading edge and resulting in downward pressures and an increased under-turning moment. The present simulation is not capturing the complicated fluid dynamics at the leading edge of the block, which is likely why the overestimate in block stability is more pronounced for smaller thickness to flow depth ratios.

\subsection{Ice accumulation at an obstruction}

Ice jamming can cause an increase in the forces exerted on hydraulic structures. Estimating these forces is of great interest to engineers. In this simulation, the soft-sphere model is used to simulate sphere-shaped solids accumulating at an obstruction of narrowly spaced piers in an open channel. The purpose of this simulation is to examine the ice accumulation upstream of an obstruction. Further work could result in quantitative estimates of the pier loading from both the ice accumulation and the fluid flow. 
Robb, D., Gaskin, S.J., Marongiu, J-C (2016) SPH-DEM model for free surface flows containing solids applied to river ice jams, Journal of Hydraulic Research, 54(1): 27-40. doi: 10.1080/00221686.2015.1131203

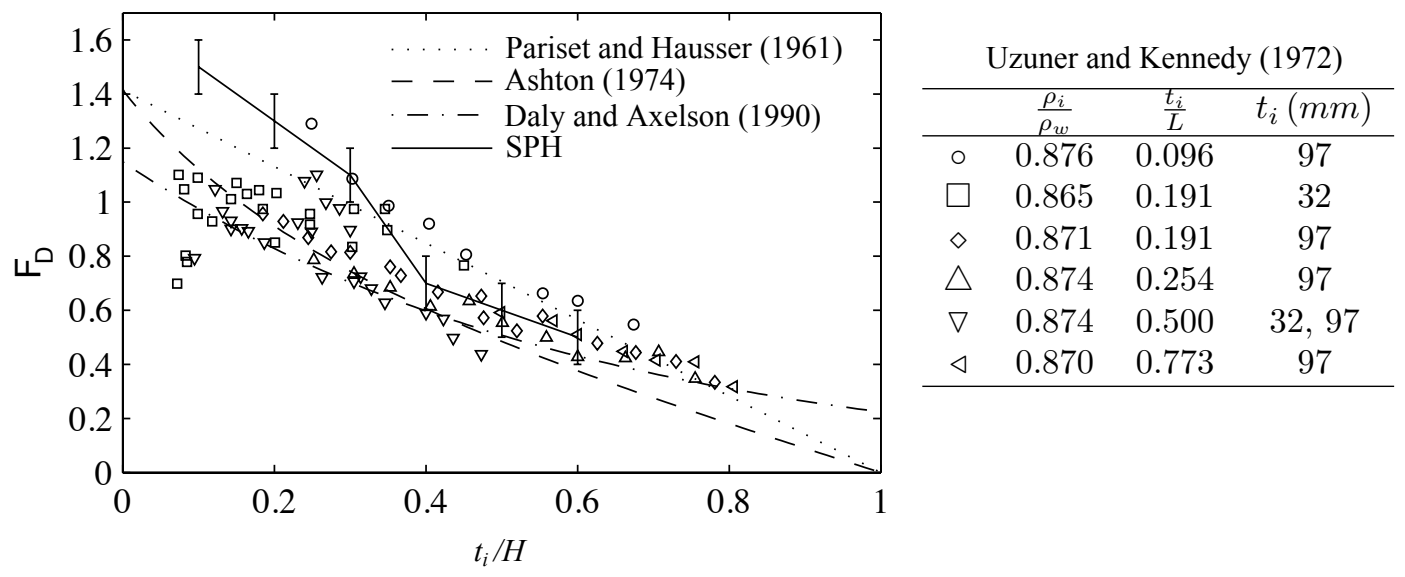

Figure 15 Floating block stability: numerical results compared to theory and experiments by Pariset and Hausser (1961), Ashton (1974), Daly and Axelson (1990), and Uzuner and Kennedy (1972)

The three-dimensional computational domain contains 600 solids located upstream of 7 vertically oriented cylindrical piles spanning a straight rectangular channel. The solids are $0.2 \mathrm{~m}$ in diameter and have a density $\rho_{i}=920 \mathrm{~kg} / \mathrm{m}^{3}$. They float partially submerged in water with a flow depth of $2 \mathrm{~m}$ and a velocity of $1 \mathrm{~m} / \mathrm{s}$. The computational domain is $17.5 \mathrm{~m}$ long, $5 \mathrm{~m}$ wide. The piers are $0.24 \mathrm{~m}$ in diameter and spaced $0.71 \mathrm{~m}$ on centre. The simulation was conducted with initial particle spacing $\Delta x=0.08 \mathrm{~m}$.

Since the solids are arranged in a single layer with a small space between each one, they are expected to initially travel at about the same speed as the water surface. As they float downstream, the solids near the channel walls are expected to slow down due to friction. As these solids decelerate, they will likely experience glancing collisions with faster moving solids farther from the wall, causing further deceleration in subsequent layers. Given enough time, the effects of the bank resistance may be felt all the way to the centreline of the channel. Under the right flow conditions and solid concentrations, the solids may form a contiguous layer and form a jam.

Figure 16 shows a time sequence of the simulation, in plan and in profile. The water has been removed while post-processing the computational results to better view the ice floes. It should be highlighted that the numerical simulation did in fact contain SPH fluid particles and the internal flow field and fluid-solid interactions were calculated in an identical way to the simulation shown previously. As expected, the bank resistance slows the solids near the walls first. They, in turn, decelerate the faster moving solids farther from the wall. As the process continues, it is apparent that the fastest moving solids are near the centreline of the channel. The bank resistance and subsequent collisions between solids cause the surface layer to resist the flow. Consequently, the flow backs up and the water level rises slightly as shown in Fig. $16 \mathrm{~b}$ and Fig. 16c; however, the solids do not form a jam. With lower water velocities, smaller pile spacing, or higher solid concentration, jam initiation may occur. Further simulations are required to explore the processes occurring over a range of parameters. 


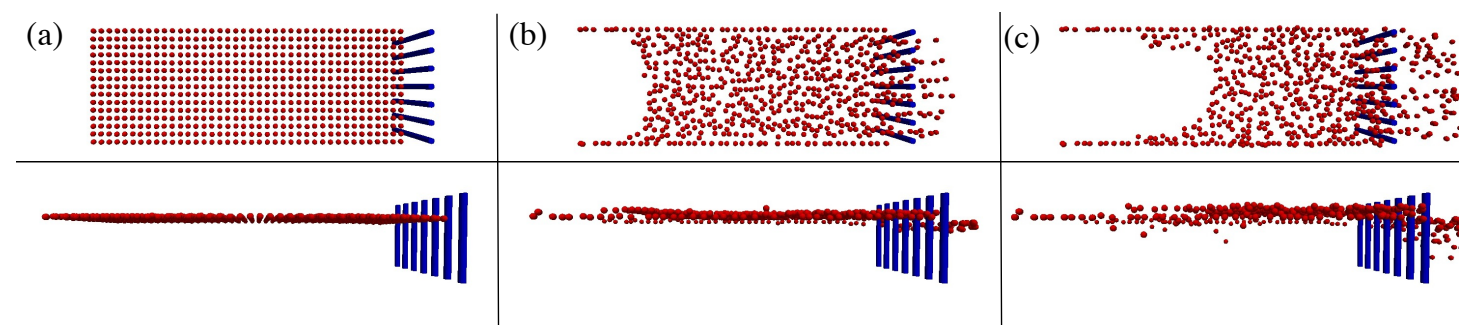

Figure 16 Ice accumulation: time sequence showing the movement of the ice floes at times (a) $0 \mathrm{~s}$, (b) $5 \mathrm{~s}$, and (c) $10 \mathrm{~s}$, plan view (top panels), elevation view (bottom panels)

\section{Discussion of results}

In recent studies presenting coupled SPH-DEM models, some of the future directions noted for further development were to use a two-way coupled SPH-DEM model (Fernandez et al., 2011), to use a three-dimensional model (Ren et al., 2013), to improve the fluid-solid boundary treatment (Canelas et al., 2013), and to include contact friction between particles (Robinson et al., 2014). This study presents a two-way coupled SPH-DEM model, where both the fluid-solid and solid-solid interactions are resolved. Simulations can be run in both two and three dimensions. The model uses a fluid-solid boundary treatment that is well suited for complex geometries and moving solid boundaries. The numerical results show that this boundary treatment can accurately predict the hydrodynamic forces exerted on a wedge entering still water and the buoyancy of a floating block. The soft-sphere model presented herein includes contact friction between particles. Numerical results of a collapse of a granular column show realistic behaviour of a granular flow through a range of flow regimes and the height of the final deposit and the run-out distance agree reasonably well with scaling laws proposed by Lajeunesse et al. (2004).

Overall, the SPH-DEM model produced the expected results for the validation test cases in section 5. The model was then applied to two simple river ice dynamics applications. The floating block stability simulations predicted the critical densimetric Froude number $F_{D}$ for under-turning satisfactorily for small block thickness to depth ratios $t_{i} / H$, but over predicted $\mathrm{F}_{\mathrm{D}}$ for larger values of $t_{i} / H$. The model is expected to give more accurate results with a more detailed treatment of viscous and turbulent effects near the leading edge of the stationary cover and the floating block. The ice accumulation at an obstruction simulation showed an application of the SPH-DEM model to predict jam initiation at an obstruction in an open channel. Although the assessment of these results is currently only qualitative, the simulation shows several characteristics common to the early stages of jam initiation. While these results require further refinement, they demonstrate the potential for this model to be a useful tool to predict ice jamming and to estimate the forces on hydraulic structures due to ice accumulation.

The present soft-sphere DEM models circular solids in two dimensions and spherical solids in three dimensions. An approach to accurately simulate three-dimensional dilated circular discs, as in Hopkins and Tuhkuri (1999) or Sun and Shen (2012), is being developed. Future work may include extending this approach to solids of arbitrary shape. The present SPH-DEM model resolves the interstitial fluid between solid particles. This approach is computationally 
Robb, D., Gaskin, S.J., Marongiu, J-C (2016) SPH-DEM model for free surface flows containing solids applied to river ice jams, Journal of Hydraulic Research, 54(1): 27-40. doi: 10.1080/00221686.2015.1131203

demanding and restricts the size of the computational domain that is feasible to model. To model larger length scales, future work may investigate a mesoscale SPH-DEM approach, where interstitial fluid between solids is unresolved and the fluid-solid interactions are coupled using drag closures as in Robinson et al. (2014).

\section{Conclusions}

A coupled SPH-DEM model for simulating free-surface fluid flows containing solids has been presented. The model's ability to simulate fluid-solid and solid-solid interactions has been demonstrated and then applied to two river ice dynamics problems. The results show the ability to simulate under-turning of a floating block approaching a stationary cover. They also exhibit several characteristics common to the early stages of ice jam initiation. A notable feature of the SPH-DEM model is that both the fluid phase with SPH and the solid phase with the DEM are modelled using particle methods. The result is an entirely meshless method to model the fluid-solid system. The method is, therefore, suitable for problems with a free surface and with complex moving geometries. River ice flows contain both these features and serve as an interesting application for the SPH-DEM approach.

\section{Acknowledgments}

The authors would like to thank Etienne Parkinson and Marc Villeneuve for their advice and contributions. D.M.R. was supported by the National Science and Engineering Research Council (Postgraduate Scholarship) and by Andritz Hydro in Vevey, Switzerland.

\section{Funding}

D.M.R. was supported by the National Science and Engineering Research Council (Postgraduate Scholarship) and by Andritz Hydro in Vevey, Switzerland. S.G. is grateful for financial support from the National Sciences and Engineering Research Council (NSERC) Discovery Grant Program.

\section{Notation}

Scalar quantities:

$a=$ aspect ratio of the initial height to the initial radius of a granular column (-)

$c_{0}=$ speed of sound $(\mathrm{m} / \mathrm{s})$

$D=$ kernel support (-)

$\mathrm{F}_{\mathrm{D}}=$ densimetric Froude number (-)

$h=$ smoothing length (m)

$h_{0, \infty}=$ initial height $\left(h_{0}\right)$ and the height of the final deposit $\left(h_{\infty}\right)$ of a granular column $(\mathrm{m})$

$k=$ spring stiffness $(\mathrm{N} / \mathrm{m})$

$L=$ length of floating block (m)

$m=\operatorname{mass}(\mathrm{kg})$ 
$N=$ number of sample points

$P=$ pressure $(\mathrm{Pa})$

$r_{0, \infty}=$ initial radius $\left(r_{0}\right)$ and final run-out distance $\left(r_{\infty}\right)$ of a granular column $(\mathrm{m})$

$t=$ time (s)

$t_{i}=$ thickness of floating block $(\mathrm{m})$

$\Delta t=$ timestep (s)

$V=$ depth-averaged upstream water velocity $(\mathrm{m} / \mathrm{s})$

$W=$ kernel function $\left(\mathrm{m}^{-3}\right.$ (in 3D))

$\Delta x=$ discretization size $(\mathrm{m})$

$\delta=$ overlap between two solids (m)

$\varepsilon=$ coefficient of restitution (-)

$\varepsilon_{L_{2}}=$ relative $L_{2}$ norm error

$\eta=$ damping coefficient (-)

$\mu=$ coefficient of friction (-)

$\omega_{a, b}=$ particle weight (units of volume in $3 \mathrm{D}$ and area in $\left.2 \mathrm{D}\right)\left(\mathrm{m}^{3}\right)$

$\mu=$ internal angle of friction $\left(^{\circ}\right)$

$\rho=$ density $\left(\mathrm{kg} / \mathrm{m}^{3}\right)$

$\phi=$ general variable

\section{Vector and tensor quantities:}

$\boldsymbol{\omega}=$ angular velocity $\left(\mathrm{s}^{-1}\right)$

$\tau=$ torque $(\mathrm{N} \cdot \mathrm{m})$

$v=$ linear velocity of a rigid body's centre of mass $(\mathrm{m} / \mathrm{s})$

$\boldsymbol{F}=$ force $(\mathrm{N})$

$\mathrm{I}=$ moment of inertia $\left(\mathrm{kg} \cdot \mathrm{m}^{2}\right)$

$\boldsymbol{n}, \boldsymbol{t}=$ unit vectors pointing normal (n) and tangent $(\boldsymbol{t})$ to a surface (-)

$\boldsymbol{p}=$ position vector on the surface of a rigid body $(\mathrm{m})$

$\boldsymbol{u}=$ velocity vector $(\mathrm{m} / \mathrm{s})$

$\boldsymbol{x}=$ position vector $(\mathrm{m})$

\section{Superscripts:}

$*=$ solution to the Riemann problem

Subscripts:

$i, j=$ quantities at the particle of interest $(i)$ and at a particle in its neighbourhood $(j)$

$i, w=$ quantities related to ice $(i)$ and water $(w)$

$e=$ experimental solution

$n=$ numerical solution 
Robb, D., Gaskin, S.J., Marongiu, J-C (2016) SPH-DEM model for free surface flows containing solids applied to river ice jams, Journal of Hydraulic Research, 54(1): 27-40. doi: 10.1080/00221686.2015.1131203

\section{References}

Ashton, G. D. (1974). Froude criterion for ice-block stability. Journal of Glaciology, 13, 974.

Batchelor, G. (1967). An introduction to fluid dynamics. Cambridge: C.U. Press.

Beltaos, S. (1993). Numerical computation of river ice jams. Canadian Journal of Civil Engineering, 20, 88-99. doi:10.1139/193-010

Canelas, R., Ferreira, R. M., Crespo, A. J., \& Domìnguez, J. M. (2013). A generalized SPHDEM discretization for the modelling of complex multiphasic free surface flows. In J.E. Olsen (Ed.), Proceedings of the 8th International SPHERIC Workshop (pp. 74-79). Trondheim: SPHERIC.

Cleary, P. W., \& Campbell, C. S. (1993). Self-lubrication for long runout landslides: examination by computer simulation. Journal of Geophysical Research, 98, 21911-21924. doi:10.1029/93JB02380

Cleary, P. W., \& Sawley, M. L. (2002). DEM modelling of industrial granular flows: 3D case studies and the effect of particle shape on hopper discharge. Applied Mathematical Modelling, 26, 89-111. doi:10.1016/S0307-904X(01)00050-6

Cundall, P. A., \& Strack, O. D. L. (1979). A discrete numerical model for granular assemblies. Geotechnique, 29, 47-65. doi:10.1680/geot.1979.29.1.47

Dalrymple, R., \& Rogers, B. (2006). Numerical modeling of water waves with the SPH method. Coastal Engineering, 53, 141-147. doi:10.1016/j.coastaleng.2005.10.004

Daly, S. F., \& Axelson, K. D. (1990). Stability of floating and submerged blocks. Journal of Hydraulic Research, 28, 737-752. doi:10.1080/00221689009499023

Dubois, F. (2001). Partial Riemann Problem, Boundary Conditions, and Gas Dynamics. In L. Tourette, \& L. Halpern (Eds.), Absorbing Boundaries and Layers, Domain Decomposition Methods: Applications to Large Scale Computer (pp. 16-77). Hauppauge, NY: Nova Science Pub.

Fernandez, J., Cleary, P., Sinnott, M., \& Morrison, R. (2011). Using SPH one-way coupled to DEM to model wet industrial banana screens. Minerals Engineering 24, 741-753. doi:10.1016/j.mineng.2011.01.004

Flato, G. M., \& Gerard, R. (1986). Calculation of ice jam profiles. In Proceedings of the 4th Workshop on the Hydraulics of Ice Covered Rivers (pp. C-3.1-C-3.25). Montreal: CRIPE.

Gotoh H., Ikari H., \& Yasuoka T. (2009). Simulation of armor blocks in front of caisson breakwater by DEM-MPS hybrid model. In J.S. Chung, S. Prinsenberg, S. W. Hong, \& S. Nagata (Eds.) Proceedings of the 19th International Offshore and Polar Engineering Conference (pp. 365-370). Osaka: ISOPE.

Gutfraind, R., \& Savage, S. B. (1998). Flow of fractured ice through wedge-shaped channels: smoothed particle hydrodynamics and discrete-element simulations. Mechanics of Materials, 29, 1-17. doi:10.1016/S0167-6636(97)00072-0

Hopkins, M. A., \& Tuhkuri, J. (1999). Compression of floating ice fields. Journal of Geophysical Research, 104, 15815-15825. doi: 10.1029/1999JC900127 
Robb, D., Gaskin, S.J., Marongiu, J-C (2016) SPH-DEM model for free surface flows containing solids applied to river ice jams, Journal of Hydraulic Research, 54(1): 27-40. doi: 10.1080/00221686.2015.1131203

Hydraulic Engineering Center (HEC). (1998). HEC-RAS river analysis system. Hydraulic Reference Manual, Version 2.2, US Army Corps of Engineers. doi: 10.1029/1999JC900127

Lacaze, L., \& Kerswell, R.R. (2009). Axisymmetric granular collapse: a transient 3D flow test of viscoplasticity. Physical Review Letters, 102, 108305. doi:10.1103/PhysRevLett.102.108305

Lajeunesse, E., Mangeney-Castelnau, A., \& Vilotte, J. (2004). Spreading of a granular mass on a horizontal plane. Physics of Fluids, 16, 2371-2381. doi:10.1063/1.1736611

Leboeuf, F., \& Marongiu, J. C. (2011). Application of SPH methods to conservative equations. In F. Magoules (Ed.) Computational fluid dynamics (pp.87-116). Boca Raton: Chapman and Hall/CRC.

Lee, B. H., Park, J. C., Kim, M. H., Jung, S. J., Ryu, M. C., \& Kim, Y. S. (2010). Numerical simulation of impact loads using a particle method. Ocean Engineering, 37, 164-173. doi:10.1016/j.oceaneng.2009.12.003

Lopez, D., Marivela, R., \& Garrote, L. (2010). Smoothed particle hydrodynamics model applied to hydraulic structures: A hydraulic jump test case. Journal of Hydraulic Research, 48(1 Extra Issue), 142-158. doi:10.1080/00221686.2010.9641255

Manenti, S., Panizzo, A., Ruol, P., \& Martinelli, L. (2008). SPH simulation of a floating body forced by regular waves. In P. Maruzewski (Ed.) Proceedings of the 3rd International SPHERIC Workshop (pp. 38-41). Lausanne: SPHERIC.

Marongiu J.C., Leboeuf F., Favre J.M., \& Parkinson E. (2006). Numerical simulation of the flow in a Pelton turbine using the meshless method SPH. Paper presented at the meeting of the Hydro 2006 Conference, Porto Carras, Greece.

Marongiu J.C., Leboeuf F., Caro J., \& Parkinson E. (2010). Free surface flows simulations in Pelton turbines using an hybrid SPH-ALE method. Journal of Hydraulic Research, 48(1 Extra Issue), 40-49. doi:10.1080/00221686.2010.9641244

Monaghan, J. J. (1994). Simulating free surface flows with SPH. Journal of Computational Physics, 110, 399-406. doi:10.1006/jcph.1994.1034

Monaghan, J. J., Kos, A., \& Issa, N. (2003). Fluid motion generated by impact. . Journal of Waterway, Port, Coastal, and Ocean Engineering, 129, 250-259. doi:10.1061/(ASCE)0733950X(2003)129:6(250)

Morris, J. P., Fox, P. J., \& Zhu, Y. (1997). Modeling low Reynolds number incompressible flows using SPH. Journal of Computational Physics, 136, 214-226. doi:10.1006/jcph.1997.5776

Nolin, S., Roubtsova, V., Morse, B., \& Quach, T. (2009). Smoothed particle hydrodynamics hybrid model of ice-jam formation and release. Canadian Journal of Civil Engineering, 36, 1133-1143. doi:10.1139/L09-051

Oger, G., Doring, M., Alessandrini, B., \& Ferrant, P. (2006). Two-dimensional SPH simulations of wedge water entries. Journal of Computational Physics, 213, 803-822. doi:10.1016/j.jcp.2005.09.004

Omidvar, P., Stansby, P. K., \& Rogers, B. D. (2012). Wave body interaction in 2D using smoothed particle hydrodynamics (SPH) with variable particle mass. International Jour- 
Robb, D., Gaskin, S.J., Marongiu, J-C (2016) SPH-DEM model for free surface flows containing solids applied to river ice jams, Journal of Hydraulic Research, 54(1): 27-40. doi: 10.1080/00221686.2015.1131203

nal for Numerical Methods in Fluids, 68, 686-705. doi:10.1002/fld.2528

Omidvar, P., Stansby, P. K., \& Rogers, B. D. (2013). SPH for 3D floating bodies using variable mass particle distribution. International Journal for Numerical Methods in Fluids, 72, 427-452. doi:10.1002/fld.3749

Pariset, E., \& Hausser, R. (1961). Formation and evolution of ice covers on rivers. Transactions, Engineering Institute of Canada 5, 41-49.

Pariset, E., Hausser, R., \& Gagnon, A. (1966). Formation of ice covers and ice jams in rivers. Journal of the Hydraulics Division, ASCE, 92(6), 1-24.

Ren, B., Jin, Z., Gao, R., Wang, Y.-X., \& Xu, Z.-L. (2014). SPH-DEM modeling of the hydraulic stability of 2D blocks on a slope. Journal of Waterway, Port, Coastal, and Ocean Engineering, 140, 04014022. doi:10.1061/(ASCE)WW.1943-5460.0000247

Robinson, M., Ramaioli, M., \& Luding, S. (2014). Fluid-particle flow simulations using twoway-coupled mesoscale SPH-DEM and validation. International Journal of Multiphase Flow, 59, 121-134. doi:10.1016/j.ijmultiphaseflow.2013.11.003

Rogers, B. D., Dalrymple, R. A., \& Stansby, P. K. (2010). Simulation of caisson breakwater movement using 2-D SPH. Journal of Hydraulic Research, 48(1 Extra Issue), 135-141. doi:10.1080/00221686.2010.9641254

Shen, H. T., Su, J. S., \& Liu, L. W. (2000). SPH simulation of river ice dynamics. Journal of Computational Physics 165, 752-770. doi:10.1006/jcph.2000.6639

Staron, L., \& Hinch, E.J. (2005). Study of the collapse of granular columns using two-dimensional discrete-grain simulation, Journal of Fluid Mechanics 545, 1-27. doi:10.1017/S0022112005006415

Stockstill, R. L., Daly, S. F., \& Hopkins, M. A. (2009). Modeling Floating Objects at River Structures. Journal of Hydraulic Engineering 135, 403-414. doi:10.1061/(ASCE)07339429(2009)135:5(403)

Sun, S., \& Shen, H. H. (2012). Simulation of pancake ice load on a circular cylinder in a wave and current field. Cold Regions Science and Technology, 78, 31-39. doi:10.1016/j.coldregions.2012.02.003

Toro, E. F. (2009). Riemann solvers and numerical methods for fluid dynamics: a practical introduction. Berlin, Heidelberg: Springer Verlag.

Uzuner, M. S., \& Kennedy, J. F. (1972). Stability of floating ice blocks. Journal of Hydraulics Division, ASCE, 98, 2117-2133.

Uzuner, M. S., \& Kennedy, J. F. (1976). Theoretical model of river ice jams. Journal of Hydraulics Division, ASCE, 102, 1365-1383.

Van der Hoef, M. A., Ye, M., van Sint Annaland, M., Andrews, A. T., Sundaresan, S., \& Kuipers, J. A. M. (2006). Multiscale modeling of gas-fluidized beds. Advances in Chemical Engineering, 31, 65-149. doi:10.1016/S0065-2377(06)31002-2

Vila, J. P. (1999). On particle weighted methods and smooth particle hydrodynamics. Mathematical Models and Methods in Applied Science 9, 161-209.

doi:10.1142/S0218202599000117 
Robb, D., Gaskin, S.J., Marongiu, J-C (2016) SPH-DEM model for free surface flows containing solids applied to river ice jams, Journal of Hydraulic Research, 54(1): 27-40. doi: 10.1080/00221686.2015.1131203

Walton, O. R. (1993). Numerical simulation of inelastic, frictional particle-particle interactions. In M.C. Roco (Ed.), Particulate two-phase flow (pp. 884-911). Oxford: ButterworthHeinemann.

Zhao, R., Faltinsen, O., \& Aarsnes, J. (1997). Water entry of arbitrary two-dimensional sections with and without flow separation. In Proceedings of the 21st Symposium on Naval Hydrodynamics (pp. 408-423). Washington: The National Academies Press. 